Voix et Images

voixetimages

\title{
Le souffle du Noroît
}

\section{André Brochu}

Volume 38, numéro 1 (112), automne 2012

URI : https://id.erudit.org/iderudit/1013456ar

DOI : https://doi.org/10.7202/1013456ar

Aller au sommaire du numéro

Éditeur(s)

Université du Québec à Montréal

\section{ISSN}

0318-9201 (imprimé)

1705-933X (numérique)

Découvrir la revue

Citer ce compte rendu

Brochu, A. (2012). Compte rendu de [Le souffle du Noroît]. Voix et Images, 38(1), 144-149. https://doi.org/10.7202/1013456ar d'utilisation que vous pouvez consulter en ligne.

https://apropos.erudit.org/fr/usagers/politique-dutilisation/ 


\section{P O ÉS I E \\ Le souffle du Noroît \\ $++$}

ANDRÉ BROCHU

Université de Montréal

Les Éditions du Noroît ne cessent de manifester une admirable vitalité à travers leurs publications courantes, mais aussi les choix de poèmes ou rétrospectives qui couronnent les œuvres les plus importantes. Paul Chanel Malenfant et le regretté Michel Beaulieu sont parmi les plus récents bénéficiaires de ce genre d'hommage. Mais je parlerai d'abord d'un essai d'une rare tenue, au Noroît également, signé Jacques Brault.

\section{JACQUES BRAULT FACE AU POÈME}

Il est l'un des principaux poètes du Québec, même s'il ne jouit pas de la popularité exceptionnelle d'un Nelligan ou d'un Gaston Miron. Universitaire, il est aussi un lecteur fort érudit de la tradition poétique, depuis l'Antiquité jusqu'à nos jours en passant par le Moyen-Âge (il a écrit sur Hélinand de Froidmont et publié un choix de textes de Bernard de Clervaux), il fréquente aussi bien les classiques que les modernes, et il joint à sa vaste connaissance de la poésie occidentale celle des littératures de l'Orient, notamment la japonaise. De plus, sa formation de philosophe fait de lui un fin connaisseur des principaux commentateurs de la tradition poétique. La pratique et le savoir du poème, chez Jacques Brault, sont tout à fait remarquables, et un bref mais substantiel essai, Dans la nuit du poème ${ }^{1}$, résume la méditation de toute sa vie sur cet étrange objet de langage dont très peu d'écrivains ont prétendu cerner les contours.

Toutefois, Jacques Brault ne cherche pas à cerner une définition du poème, non plus que du rythme qui en serait l'élément fondateur. Sa connaissance du langage poétique, qui s'appuie sur la référence à un grand nombre d'auteurs (plus de soixantedix sont mentionnés en moins de cinquante pages), ne cumule concepts et intuitions que pour se saborder, en fin de compte, et faire apparaître l'impossibilité de dire ce qui se dit en poésie. La plupart des grandes voix citées, qui ont énoncé les perspectives les plus profondes sur le poétique, le plus souvent pour se récuser et substituer

$$
+++
$$

1 Jacques Brault, Dans la nuit du poème, Montréal, Éditions du Noroît, coll. «Chemins de traverse», 2011, 52 p. 
l'humour à la dissertation, forment l'extraordinaire caisse de résonance de la réflexion de Brault. Ces auteurs sont très souvent français et relativement récents, tels Stéphane Mallarmé, Arthur Rimbaud, Paul Claudel, Paul Valéry ou, plus près de nous encore, André Breton, Louis Aragon, Henri Michaux, Francis Ponge, Raymond Queneau, Philippe Jaccottet, Jacques Réda et Jacques Roubaud. Bien entendu, sont aussi appelés à la barre les parangons de la modernité, Rainer Maria Rilke, Octavio Paz et Paul Celan, dont les précieuses réalisations élèvent la littérature à des hauteurs inégalées.

Les Québécois, dans tout cela? On en trouve de rares mentions, et la plus voyante, celle de Robert Marteau, chevauche les littératures de France et d'ici. N'y voyons nul mépris, de la part de celui qui nous a procuré la précieuse édition critique d'Hector de Saint-Denys Garneau (réalisée avec Benoît Lacroix), une présentation d'Alain Grandbois dans la collection «Poètes d'aujourd'hui», un texte fondateur, sous forme de conférence, sur "Miron le magnifique», etc. Il est sans doute trop tôt pour faire la synthèse des réflexions de nos écrivains sur la nature du poème, mais on peut savoir gré à Jacques Brault de s'être adressé à des sources fiables et universellement connues, et d'avoir tenté d'en dégager l'enseignement.

Le grand nombre d'écrivains cités pourrait signifier l'impossibilité de prendre parti devant la multiplicité des opinions qui ont cours. Il s'agit plutôt d'un trait caractéristique de la démarche de Jacques Brault. Comme poète, par exemple, son inspiration d'un recueil à l'autre apparaît fort variée. On chercherait en vain un thème sousjacent à l'ensemble, voire à des segments étendus de sa production. Pourtant, la plus grande rigueur préside à l'élaboration de ses poèmes, même de ceux qui manifestent une certaine désinvolture (on pense à un titre comme «Si la poésie sacre le camp ${ }^{2} »$ ). Mais les nombreux aspects de la vie sollicitent tour à tour son esprit tourné vers tout ce qui réclame d'être dit. Quoi de plus différent, poétiquement, que le lyrisme de Mémoire ${ }^{3}$ et ces vers de La poésie ce matin ${ }^{4}$ :
ça va bon train ça va/savoir tu
ne peux pas/droit et de fer le
chemin passe sur les dormants/et
s'enfuit le sang (192)

ou que ces lignes d'Au bras des ombres ${ }^{5}$ :

Où fragment d'un cauchemar tu te pulses sentier qui a du chiendent aux pieds qu'est-ce que tu broussailles en pleine ville ça mugit ça trémousse (58)

2 Premier poème du recueil intitulé La poésie ce matin, réédité dans Jacques Brault, Poèmes, Montréal, Éditions du Noroît, 2000, coll. «Ovale», p. 121. 3 Jacques Brault, Poèmes, p. 21-111. 4 Jacques Brault, Poèmes, p. 113-217. 5 Jacques Brault, Au bas des ombres, Paris/Saint-Hippolyte, Arfuyen/Éditions du Noroît, coll. «Résonance», 1997, 72 p. 
et ces vers de L'artisan ${ }^{6}$ :

Monde d'avant que tu es beau n'étant plus quelle mort antérieure dans le fil

des lianes pousse un poulpe

branchu au magma volubile à l'obscur qui s'affale (75)

L'expérimentation continuelle du poète est en harmonie avec, ici, sa recherche intellectuelle de la nature du poème; nature rendue encore plus problématique de nos jours, moins par le vers libre que par le poème en prose, dénué des caractéristiques formelles qui le distingueraient de la prose proprement dite (17). Malgré l'impossibilité des définitions, sa réflexion amène Jacques Brault à montrer l'étroite collaboration, en poésie, entre le vers et la prose:

\footnotetext{
Que la prose fasse irruption dans le souffle versifiant, et voilà qu'elle déplie le vers, qu'elle le démesure en partie et le sauve de sa maîtrise ronronnante. N'est-ce pas ce qu'a fait Baudelaire, puis Queneau, et ce que fait Réda? À l'inverse, que le vers se jette en travers de la prose, qu'il lui coupe son allant, qu'il l'essouffle un peu, et la bavarde apprendra le prix du silence. (30-31)
}

On aura une idée de la riche problématique de Jacques Brault en lisant la définition minimale qu'il avance enfin de la poésie, non pas « essence éternelle» mais « vibration hétérochronique où se distribueraient eurythmiquement le phonétique, l'accentuel, le syntaxique, le sémantique, le graphique, etc.» (39) Quant au rythme - peut-être «l'élément inaugural du poème» (45), mais ce n'est pas sûr -, il opère au plus noir de la nuit du texte.

Nous sommes sans doute en présence ici d'une poétique négative, comme il y a la théologie négative, mais Jacques Brault fait audacieusement le tour des questions les plus fondamentales qui se posent à propos de la poésie, et il y répond, en silence si l'on peut dire, avec l'ardente circonspection d'un penseur autant que d'un praticien du haut langage.

\section{MER ET PIERRES DE MALENFANT}

Après avoir signé de précieux choix de poèmes et des présentations d'auteures aussi différentes que Simone Routier, Nicole Brossard et Claudine Bertrand, Louise Dupré (Grand Prix Québécor du Festival international de poésie 2011 et Prix du Gouverneur Général 2011 pour son dernier recueil ${ }^{7}$ ) offre au public le choix de poèmes de Paul Chanel Malenfant qu'elle vient de réaliser ${ }^{8}$. La grande qualité d'une œuvre appréciée

6 Jacques Brault, L'artisan, Montréal, Éditions du Noroît, 2006, 124 p. 7 Louise Dupré, Plus haut que les flammes, Montréal, Éditions du Noroît, 2010, 112 p. 8 Paul Chanel Malenfant, Traces de l'éphémère, choix de poèmes et présentation de Louise Dupré, Montréal, Éditions du Noroît, coll. «Ovale », Montréal, 2011, 256 p. 
des connaisseurs, mais pas toujours présente à l'avant-scène, est ainsi soulignée. De 1976 à nos jours, le poète rimouskois (qui n'est rien moins que régionaliste, soulignons-le, mais qui fait corps avec son pays de pierre et d'eau) a publié une vingtaine de recueils, souvent considérables. Une grande unité de thèmes et d'impressions émane de cette production, même si certains sujets prennent place plutôt vers son début, son milieu ou sa fin. Par exemple, c'est dans l'un des tout derniers recueils que le thème de l'amitié amoureuse se dévoile alors que, présent bien plus tôt, il s'énonçait jusque-là de plus discrète façon.

Le pays impose dès le premier recueil ses vastes perspectives, et il s'agit avant tout de la mer, comme l'affirme le titre: Poèmes de la mer pays. Un rapport à la nature est posé, qui sera approfondi tout le long de l'œuvre, et la réalité humaine, le plus souvent familiale, viendra s'y inscrire naturellement, avec ses bonheurs et ses drames. Les figures de la mère et du père ont une importance primordiale. Du père procède peut-être une orientation problématique: «L'adolescent interroge l'androgynie du géniteur. » (65) Quant à la mère, elle manifeste une exubérance de tout le corps: «ne regarde pas le ventre de ta mère qui danse et jouit et hurle chienne en chaleur et si belle dans le frisson et le spasme et le cri à la musique fondus» (71). La congruence de la nature et de l'humain est d'autant plus grande que des homophonies les cautionnent: mer et mère, selon le rapprochement bien connu, mais aussi, pierre et père, la pierre (motif surabondant des poèmes) étant le centre compact d'un espace et du réel, qui trouve finalement son emplacement au-dessus du père, dans le rite de la mort: «cette pierre n'a pas de modèle et dans le lent étonnement de cette pierre, là où le père, dessous, je m'étonne que cela soit aussi ton nom, l'interminable fin d'un amour à n'en plus finir» (114). On peut comprendre que pierre est à la fois une sorte d'homonyme imparfait du père et le nom de l'amant. La mer, surface infinie, et la pierre d'une «insondable fixité » (147) sont des opposés et des complémentaires pour l'imaginaire, tout comme père et mère définissent ensemble et contradictoirement la destinée de l'enfant. Le langage ne cesse de les réunir et de suggérer ainsi leur complicité :

\author{
Matin de mer après la pluie: \\ les pierres apparaissent \\ sur la présence du monde (189; je souligne.)
}

Ici, non seulement mer et pierres sont mis en relation, mais ils se prolongent dans tout l'espace signifiant par la prolifération des $m$ et des $p$, qui font surgir en sourdine le couple émotionnel du père et de la mère.

À côté de ces thèmes naturels et humains, on en trouve d'autres qui concernent de plus près l'intimité charnelle du poète, comme la peau, qui établit la démarcation entre l'intérieur et l'extérieur et fonde l'ordre du sensible, non sans relation d'ailleurs avec la pensée : «Sensibles les mots passent par la pensée de la peau » (132) - notons ici aussi l'abondance des $p$, représentative de tout un travail sur le langage qui recourt aux homophonies pour suggérer des sens adventices et éclairants.

Paul Chanel Malenfant accorde une grande place à la représentation de l'espace, surtout celui de la nature, et celui-ci ne serait pas complet sans la présence des 
couleurs par lesquelles s'affirment choses et objets. Deux couleurs surtout prédominent: le bleu (comme chez Fernand Ouellette, mais avec un sens moins spirituel) et le rouge. Le bleu est surtout indice de beauté matérielle, et l'autre, de passion. «Tout ce bleu à la place de ma mère, tout ce bleu et qui m'engloutit: jeûne et ce vertige des lettres grecques. » (66) L'espace se fait ici tout intérieur. Le rouge est associé «aux eaux d'enfance de la rivière Rouge » (173), ou encore à « des cœurs de rouge à lèvres et de buée» (120), participe de l'effusion des forces vives.

Tout en consignant en écriture les constantes de sa vie, le poète se montre très sensible aux vérités profondes de la mort, laquelle a frappé nombre d'êtres chers. Le père, la mère d'abord atteinte de la maladie d'Alzheimer, des membres plus jeunes de la famille et, enfin, l'ami aimé: autant de victimes d'un Dieu impitoyable («et je confonds le mot Deuil/avec le nom de Dieu», 168).

On admire l'étendue du registre poétique de Paul Chanel Malenfant, en grande partie autobiographique certes, mais qui, à partir de la condition personnelle profondément assumée, propose un imaginaire d'une saisissante beauté. Le chantre de la mort est aussi, avant tout, celui des émerveillements : «Un paon sur la mer. Des étincelles de pluie illuminent les visages en larmes. Tu éveilles le vent pour un vol de colombes. » (214)

\section{RÉTROSPECTIVES}

C'est une rétrospective un peu particulière que le Noroît consacre à Michel Beaulieu ${ }^{9}$ puisqu'elle rassemble, sur une étendue de moins de dix ans, les cinq recueils publiés par le poète chez cet éditeur. L'unité du corpus peut sembler problématique vu qu'elle est déterminée par référence à la maison d'édition alors que d'autres recueils, pendant la même période, ont paru ailleurs, notamment à l'Hexagone et chez Cul-Q. Mais l'inspiration du poète manifeste une continuité évidente, de l'un à l'autre des recueils réunis.

Peu de temps avant la parution de "FM - Lettres des saisons III», qui inaugure la rétrospective, la revue Études françaises décernait son prix à Michel Beaulieu pour Variables ${ }^{10}$, ce qui contribua beaucoup à faire connaître l'auteur. Or le directeur de la revue, Georges-André Vachon, intitulait judicieusement sa postface : «Qu'est-ce que cela veut dire?» (93-99), citait quelques passages dont un grand nombre de détails soulevaient des questions de compréhension. Il concluait que la poésie de Variables n'est «ni descriptive, ni expressive», qu'elle est «TRAVAIL» et, par là, "CONTREDESTIN» (99).

Cette bénéfique difficulté à comprendre le texte, elle se manifeste à la lecture de bon nombre des Poèmes réunis par le Noroît. Certes, la dimension du quotidien est omniprésente chez Beaulieu, et devrait favoriser une approche aisée du texte. Il

9 Michel Beaulieu, Poèmes (1975-1984), Montréal, Éditions du Noroît, coll. «Ovale», 2011, 334 p. 10 Michel Beaulieu, Variables, Montréal, Presses de l'Université de Montréal, coll. du «Prix de la revue Études françaises», $1973,112 \mathrm{p}$. 
arrive d'ailleurs que l'évocation de l'immédiat sombre dans la trivialité, ne laissant plus guère de place au mystère : «cette envie de pisser qui te prend tout à coup/bientôt il faudra filtrer le café/peut-être une cuillerée de poudre instantanée» (19). Ici, des éléments tout à fait transparents de la réalité sont mis bout à bout, mais ne résolvent pas pour autant le mystère du vécu.

À vrai dire, le côté indécidable de l'expression dont parle Georges-André Vachon n'a rien d'invariable et peut être tantôt assimilé à l'arbitraire («qu'elle est longue à passer les reins/dans notre monde ensemencé/la pierre/quand se fait rachitique la naissance/dans les ruelles du non-sens» [75]); tantôt, au contraire, à une touchante et complexe conscience d'exister:

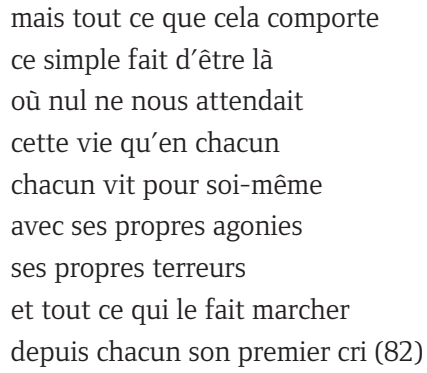

Il y a, chez Beaulieu, du meilleur et du problématique, et il y a surtout un prodigieux besoin de se réaliser dans et par la poésie, en assumant paradoxalement toute la quantité possible de réel ${ }^{11}$.

Je signale, pour finir, la réédition d'une rétrospective de France Théoret, revue et augmentée.

Bloody Mary 12, suivi de Vertiges, Nécessairement putain, Intérieurs et Étrangeté, l'étreinte, a paru d'abord en 1991, sans le dernier titre qui date de 1992.

L'intégrité, la véhémence rimbaldienne, parfois une vulgarité calculée (dans Nécessairement putain), la sensibilité aussi de cette voix unique propulsent la vision de la poète, féministe et ouverte à tout l'humain, au plus haut de l'écriture.

11 On trouvera dans André Brochu, Tableau du poème, Montréal, XYZ éditeur, 1994, p. 67-69, un compte rendu des principales sections de Poèmes: Visages et Kaléidoscope. 12 France Théoret, Bloody Mary, Montréal, TYPO, 2011, $264 \mathrm{p}$. 